J. Clin. Chem. Clin. Biochem.

Vol. 19, 1981, pp. 987-996

\title{
Wirkung einer Nembutalnarkose auf den Stoffwechsel von Noradrenalin im Gehirn der Ratte
}

\author{
Von G. Köster und H. Breuer \\ Institut für Klinische Biochemie und Biochemische Abteilung der Neurochirurgischen Universitätsklinik Bonn
}

(Eingegangen am 6. Oktober 1980/7. April 1981)

Zusammenfassung: Es wird eine spezifische und empfindliche Methode zur Trennung und Bestimmung der Metaboliten von radioaktiv markiertem Noradrenalin aus kleinsten Mengen von Hirngewebe beschrieben. Das Verfahren liefert reproduzierbare Werte; erlaubt die direkte Bestimmung aller wichtigen Noradrenalin-Metaboliten aus demselben Extrakt und eignet sich wegen seines relativ geringen Arbeitsaufwandes für größere Versuchsreihen.

Mit Hilfe dieser Methode wurde untersucht, in welcher Weise die Halbwertszeit und der Stoffwechsel von Norad renalin im Gehirn der Ratte durch eine Nembutalnarkose beeinflußt werden. Es zeigte sich, daß in Nembutalnarkose sowohl die Halbwertszeit als auch der Stoffwechsel von Noradrenalin in Hypothalamus, Thalamus sowie Pons und Medulla verlangsamt sind; darüberhinaus ist die Abgabe von Noradrenalin-Metaboliten an das Blut verzögert. Bei Stoffwechseluntersuchungen mit Neurotransmittern ist zu berücksichtigen, daß eine Nembutalnarkose sowohl das Metabolitenmuster von Noradrenalin als auch dessen zeitliche Änderungen beeinflußt.

\section{Effect of anaesthesia on the metabolism of noradrenaline in rat brain}

Summary: A specific and sensitive method is described for the separation and determination of metabolites of radioactively labelled noradrenaline in very small amounts of brain tissue. This method gives reproducible results, it allows the direct determination of all major metabolites of noradrenaline from the same extract, and is suitable for quantitating a large number of samples in a relatively short time.

This method was used to investigate how the half-life time (turnover) and the metabolism of noradrenaline in rat brain may be influenced by anaesthesia with nembutal. It was found that both the half-life time as well as the metabolism of noradrenaline are reduced during nembutal anaesthesia; in addition, the release of metabolites of noradrenaline into the blood is delayed. When studying the metabolism of neurotransmitters, it has to be considered that nembutal-narcosis influences the metabolic pattern of noradrenaline as well as its temporal changes.

\section{Einführung}

Im Vèrlauf unserer Studien über den Stoffwèchsel von Catecholaminen im Gehirn und seine Beeinflussung durch Sexualhormone (1-3) ergab sich die Notwendigkeit, in vivo-Untersuchungen an der Ratte durchzuführen und zu diesem $Z$ weck durch intraventrikuläre Injektion von $\left[{ }^{3} \mathrm{H}\right]$ Noradrenalin die Neurotransmitterpools zu markieren. Die für diese Untersuchungen nötigen Informationen konnten der Literatur nicht in ausreichendem Maß̉e entnommen werden. Deshalb wurden Vorarbeiten durchgeführt, die folgenden $\mathrm{Zwe}$ wen dienten:

(1) Entwicklung einer spezifischen und empfindlichen Methode zur Trennung und damit zur Bestimmung der Metaboliten von markiertem Noradrenalin aus kleinsten Mengen von Hirngewebe;

(2) Klärung des Einflusses einer Narkose und der Versuchsdauer auf den Stoffwechsel und das Metabolitenmuster von Noradrenalin.
In der vorliegenden Arbeit wird eine für die Aufarbeitung von Hirngewebeextrakten geeignete Modifikation der Methode von Graefe et al. (4) beschrieben, mit deren Hilfe alle Metaboliten von Noradrenalin voneinander getrennt und quantitativ bestimmt werden können. Darüberhinaus wird gezeigt, daß Nembutal nicht nur - wie bereits bekannt (5) - die Halbwertszeit von Noradrenalin verändert, sondern auch das Metabolitenmuster in Abhängigkeit von der Zeit beeinflußt.

\section{Methodik}

Noradrenalin und Metaboliten

$D, L-\left[7-{ }^{3} \mathrm{H}\right]$ Noradrenalin-hydrochlorid (spez. Aktivität $370 \mathrm{GBq} /$ mmol) wurde von Amersham-Buchler, Braunschweig, bezogen und dünnschichtchromatographisch auf Reinhcit geprüft. Noradrenalin-hydrochlorid wurde von Fluka (Buchs, Schweiz) bezogen, Normetanep hrin, 3-Methoxy-4-hydroxypheny lglykol und 3,4-Dihydroxyphenylglykol von Sigma (St. Louis, Missouri, U.S.A.), 3,4-Dihy droxy mandelsäure von EGA (Steinhcim) und Vanillinmandelsäure von Fluka (Buchs, Schıciz). 


\section{Chemikalien}

Folgende lonenaustauscher wurden benutzt: Dowex 50W-X4 ( $\mathrm{H}^{+}$-Form, 200-400 mesh; Serva, Heidelberg), Dowex AG1-X2 (Cl-Form, 200-400 mesh) und Bio Rex 5 (CI-Form, 200400 mesh), beide Bio-Rad (Richmond, California, U.S.A.). $\mathrm{Al}_{2} \mathrm{O}_{3}$ (Aktivitätsstufe II-III) stammte von Merck (Darmstadt). Nembutal wurde als Injektionslösung $(60 \mathrm{~g} / \mathrm{l})$ von Abbott (Ottignies, Belgien) bezogen, Äther pro narcosi von Hoechst (Frankfurt). Alle übrigen Chemikalien waren von p. A.-Reinheitsgrad (Merck, Darmstadt). $n$-Butanol wurde ohne vorherige Destillation benutzt.

Tiere

Männliche Wistar-Ratten mit einem Gewicht von $250-300 \mathrm{~g}$ (Iv anovas; Kisslegg/Allg.) erhielten Standardfutter (Altromin) und Wasser ad libitum.

\section{Durchführung der Narkose}

0,21-0,25 ml der wäßrigen Nembutal-Lösung (Nembutal entsprechend $50 \mathrm{mg} / \mathrm{kg}$ Körpergewicht) wurden intraperitoneal injiziert. Während der gesamten Versuchsdauer bis zu $4 \mathrm{~h}$ wurde die Nembutalnarkose aufrecht erhalten. Bei einer Versuchsdauer von mehr als einer Stunde war eine Nachinjektion von Nembutal notwendig. Die durchschnittliche Gesamtdosis betrug nach $1 \mathrm{~h}$ 56 , nach 2 h 68 , nach 3 h 81 und nach 4 h 87 mg/kg Körpergewicht. Die Äthernarkose wurde in üblicher Weise eingeleitet und sofort nach der Injektion beendet.

\section{Intraventrikuläre Injektion}

20 min nach i p. Injektion von Nembutal bzw. 5 min nach Beginn der Äthernarkose wurden $5 \mu$ l einer wäßrigen $\left[7-{ }^{3} \mathrm{H}\right]$ Noradrenalin-hydrochlorid-Lö sung (enthaltend $185 \mathrm{KBq}$ ) in den linken Seitenventrikel nach der Methode von Noble et al. (6) injiziert. 0,5 oder 1, 2, 3 oder $4 \mathrm{~h}$ nach der intraventrikulären Injektion wurden die Tiere durch Dekapitation getötet. Die Tiere, denen Noradrenalin in Äthernarkose injiziert worden war, dienten gegenüber den mit Nembutal behandelten Tieren als Vergleichsgruppe für den Wachzustand. Dieses Vorgehen erscheint gerechtfertigt, denn Schanberg et al. (10) haben keinen Einfluß der Äthernarkose auf den Stoff wechsel von Noradrenalin gefunden.

\section{Aufarbeitung des Gewebes}

Unmittelbar nach dem Dekapitieren wurde das Gehirn im Kühlraum entnommen; folgende Regionen wurden präpariert: Hypo- thalamus, Thalamus, Pons mit Medulla, Septum, Hippocampus und Cortex. Sie wurden sofort in 1-2 ml eiskalte Essigsäure $(0,5 \mathrm{~mol} / 1)$ gelegt, die folgende Zusätze enthielt: Ascorbinsäure $(5 \mathrm{~g} / \mathrm{l}), 10 \mu \mathrm{g}$ Noradrenialin und jeweils $10 \mu \mathrm{g}$ der nicht-markierten Metaboliten von Noradrenalin als Carrier. Nach dem Wiegen des Gewebes wurde im Glashomogenisator homogenisiert und das Homogenat $30 \mathrm{~min}$ bei $5000 \mathrm{~g}$ zentrifugiert. Das Sediment wurde mit $1 \mathrm{ml} \mathrm{H}_{2} \mathrm{O}$ gewaschen und erneut zentrifugiert. Die wäßrigen Überstände wurden vereinigt und mit $\mathrm{H}_{2} \mathrm{O}$ auf $3 \mathrm{ml}$ aufgefüllt. Zur Bestimmung der Gesamtaktivität wurden $50 \mu$ Aliquote mit $4 \mathrm{ml}$ Unisolve 1 versetzt. Die wäßrigen Lösungen wurden bis zur weiteren Aufarbeitung eingefroren und bei $-20^{\circ} \mathrm{C}$ aufbewahrt. Das nach Präparation der oben aufgefuihrten Hirnregionen verbliebene Gewebe wurde analog aufgearbeitet.

\section{Auftrennung der Noradrenalin-Metaboliten}

Da nicht alle Metaboliten von Noradrenalin mit Hilfe von $\mathrm{Al}_{2} \mathrm{O}_{3}$ und Ionenaustauschern in einem Arbeitsgang getrennt werden konnten, wurden zwei separate Trennungsgänge durchgeführt:

Trennungsgang I diente zur Bestimmung der Verbindungen mit Catecholstruktur (Noradrenalin, 3,4-Dihy droxypheny lglykol, 3,4-Dihy droxy mandelsäure) sowie von Normetanephrin;

Trennungsgang II diente zur Bestimmung von 3-Methoxy-4hydroxyphenylglykol, Vanillinmandelsäure sowie der Sulfate von 3,4-Dihydroxy- und 3-Methoxy-4hydroxypheny lglykol.

\section{Trennungsgang $I$}

Der Trennungsgang I (s. Schema 1) folgt der Vorschrift von Graefe et al. (4). Nach dem Auftauen wurden die essigsauren Gewebeextrakte $30 \mathrm{~min}$ bei $6000 \mathrm{~g}$ zentrifugiert, um ausgefallenes Protein abzutrennen. $1 \mathrm{ml}$ des klaren Überstañdes wurde mit $1,5 \mathrm{ml}$ Tris-HCl-Puffer $(0,05 \mathrm{~mol} / \mathrm{l}, \mathrm{pH} 8,5)$ versetzt. Extrakte von Gewebeproben von mehr als $40 \mathrm{mg}$ wurden zunächst mit Natronlauge $(1 \mathrm{~mol} / \mathrm{l})$ auf $\mathrm{pH} 6$ gebracht und dabei ausflockendes Protein erneut abzentrifugiert. Überstände bzw. übrige Proben wurden mit Natronlauge ( $1 \mathrm{~mol} / 1$, zur Feineinstellung stärker verdünnt) am $\mathrm{pH}$-Meter auf $\mathrm{pH} 8,1-8,3$ gebracht. Anschließend wurde die Analysenlösung (2,5-2,6 ml) zur Adsorption der Catechole auf eine $\mathrm{Al}_{2} \mathrm{O}_{3}$-Säule gebracht (200 mg $\mathrm{Al}_{2} \mathrm{O}_{3}$, nach Anton \& Sayre (7) HCl-gewaschen, reaktiviert und unmittelbar vor Gebrauch mit $5 \mathrm{ml}$ Natriumacetatlösung $(0,2 \mathrm{~mol} / \mathrm{l}$, pH 8,2) gewaschen). Der Durchlauf tropfte auf eine.Dowex

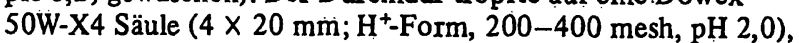
an der Normetanephrin gebunden wurde. Im Rahmen des Trennungsgangs I wurde im Durchlauf dieser Säule ohne weitere Auftrennung des Substanzgemischs lediglich die Gesamtaktivität be-

Schema 1: Trennungsgang I zur Bestimmung von Noradrenalin, Normetanephrin, 3,4-Dihydroxy mandelsäure und 3,4-Dihydroxyphenylglykol

$1 \mathrm{ml}$ Hirnextrakt $+1,5 \mathrm{ml}$ Tris-Puffer; $\mathrm{pH} 8,3$

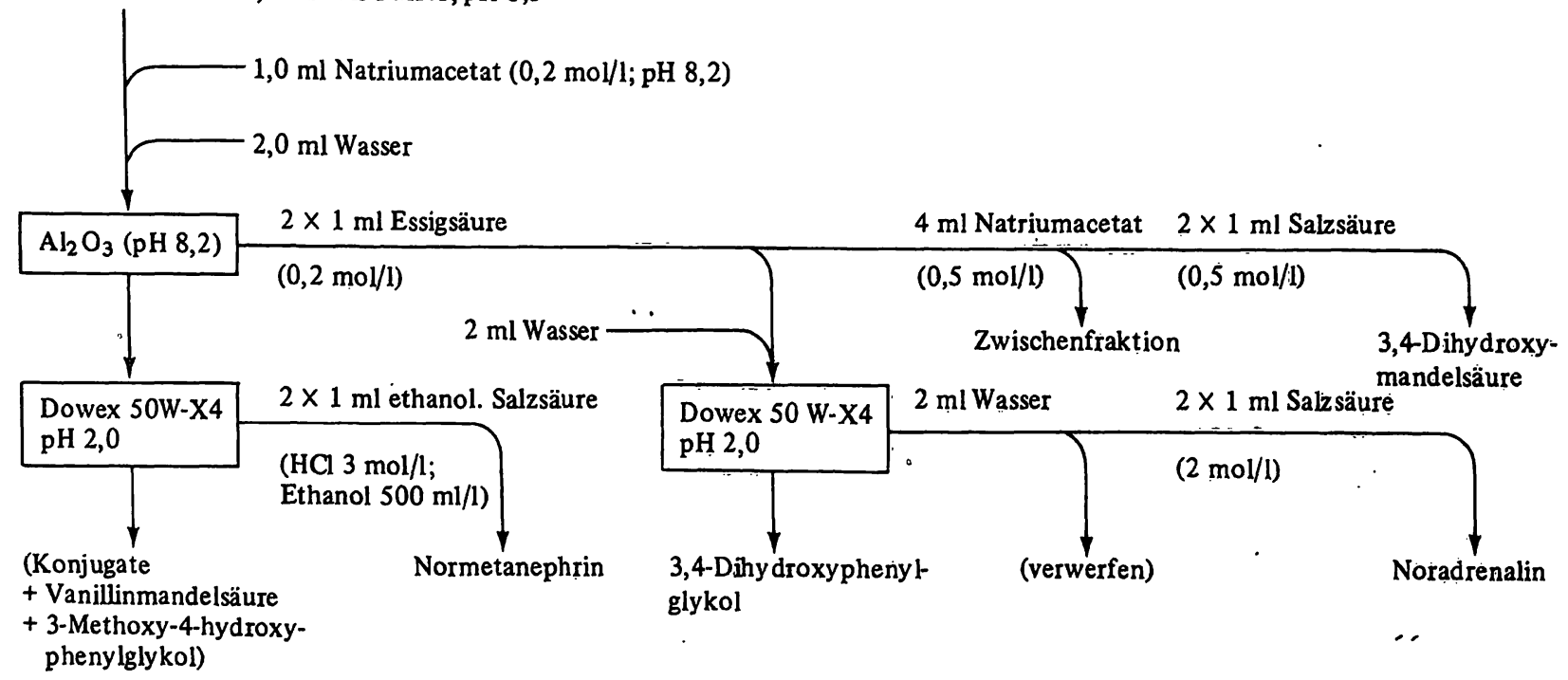


stimmt. Die $\mathrm{Al}_{2} \mathrm{O}_{3}$-Säule wurde zunächst mit $1 \mathrm{ml} \mathrm{Natrium-}$ acetat-Lösung $(0,2 \mathrm{~mol} / \mathrm{h}, \mathrm{pH} 8,2)$ und anschließend mit $2 \mathrm{ml}$ $\mathrm{H}_{2} \mathrm{O}$ gewaschen; die Waschflüssigkeit wurde direkt auf die Dowex SOW-X4-Säule geleitet. Die Elution der $\mathrm{Al}_{2} \mathrm{O}_{3}$-Säule sowie der Dowex 50W-X4-Säule erfolgte nach den Angaben von Graefe et al (4). Noradrenalin und 3,4-Dihydrophenylglykol werden dabei mit Essigsäure $(0,2 \mathrm{~mol} / \mathrm{l})$ gemeinsam von $\mathrm{Al}_{2} \mathrm{O}_{3}$ eluiert und mit Hilfe einer zweiten Dowex 50W-X4-Säule voncinander getrennt. Normetanephrin und 3,4-Dihydroxymandelsäure werden einzeln eluiert.

\section{Trennungsgang II}

Im Durchlauf der Dowex 50W-X4-Säule (vgl Trennungsgang I) befanden sich die Glykolsulfate, 3-Methoxy-4hydroxyphenyl glykol und Vanillinmandelsäure, deren Bestimmung aus dieser Lösung nur mit Schwierigkeiten durchführbar gewesen wäre. Deshalb wurde ein Teil des essigsauren Gewebeextraktes nach der Zentrifugation (siehe oben) einem getrennten Aufarbeitungsgang (Trennungsgang II, s. Schema 2) unterworfen. Dieser Trennungsgang begann mit der Adsorption der Glykolsulfate an Bio Rex 5 nach der Vorschrift von Stone \& Mendlinger (8). 1,5 ml des Gewebeextraktes wurden mit $1 \mathrm{ml} \mathrm{H} \mathrm{H}_{2} \mathrm{O}$ versetzt und mit $\mathrm{HCl}(1 \mathrm{~mol} / \mathrm{l})$ am pH-Meter auf pH 1,8 eingestellt. Anschließend wurde die Analy senlösung $(2,5-2,6 \mathrm{ml})$ zur Adsorption der Glykolsulfate auf eine Bio Rex 5-Säule ( $4 \times 20 \mathrm{~mm}$, CГ-Form, 200-400 mesh, pH 1,8) gebracht. Die Säule wude mit $2 \times 1 \mathrm{ml}$ $\mathrm{H}_{2} \mathrm{O}$ gewaschen, der Durchlauf nach Einstellung auf pH 5,5-6 eingefroren und bis zur weiteren Aufarbeitung bei $-20^{\circ} \mathrm{C}$ gelagert. 3-Methoxy-4hydroxypheny glykolsulfat wurde von der
Bio Rex 5-Säule mit $14 \mathrm{ml}$ Natriumacetat-Lösung $(0,15 \mathrm{~mol} / \mathrm{l}$, pH 6,0, gesättigt mit $n$-Butanol) eluiert. Anschließend wurde 3,4-Dihydroxyphenylglykolsulfat mit $14 \mathrm{ml}$ Natriumacetat-Lösung $(0,3 \mathrm{~mol} / \mathrm{l}, \mathrm{pH} 8,0$, gesättigt mit $n$-Butanol) eluiert. Der Durchlauf der Bio Rex 5-Säule wurde nach dem Auftauen zentrifugiert, um ausgefallenes Eiweiß abzutrennen. Dann wurde der Überstand mit Natronlauge ( $1 \mathrm{~mol} / \mathrm{l}$ ) auf $\mathrm{pH} 8,1-8,3$ eingestellt und die Lösung zur Adsorption der Catechole auf eine $\mathrm{Al}_{2} \mathrm{O}_{3}$-Säule (vgl. Trennungsgang I) gebracht. Der Durchlauf tropfte auf eine Dowex 50W-X4Säule (vgl. Trennungsgang I), an der Normetanephrin gebunden wurde. Der Durchlauf der Dowex 50W-X4-Säule, der 3-Methoxy-4hydroxypheny lglykol und Vanillinmandelsäure enthielt, wurde zur Adsorption der Vanif linmandelsäure auf eine Dowex AG1-X2-Säule $\left(4 \times 30 \mathrm{~mm}, \mathrm{Cl}^{-}\right.$Form, 200-400 mesh, pH 4,5) geleitet; im Durchlauf dieser Säule befand sich 3-Methoxy-4hydroxypheny lglykol. $\mathrm{Die} \mathrm{Al}_{2} \mathrm{O}_{3}$ Säule wurde, wie in Trennungsgang I, nacheinander zweimal mit $1 \mathrm{ml}$ Essigsäure $(0,2 \mathrm{~mol} / \mathrm{l})$. einmal mit $4 \mathrm{ml}$ Essigsäure $(0,5 \mathrm{~mol} / \mathrm{l})$ und zweimal mit $1 \mathrm{ml} \mathrm{HCl}(0,5 \mathrm{~mol} / \mathrm{l})$ eluiert; die Dowex $50 \mathrm{~W}$ X4-Säule wurde zweimal mit $1 \mathrm{ml}$ alkoholischer $\mathrm{HCl}(3 \mathrm{~mol} / \mathrm{l}$ $\mathrm{HCl}$ in $500 \mathrm{ml} / \mathrm{l}$ Ethanol) eluiert. Von der Dowex Ag1-X2-Säule wurde die Vanillinmandelsäure mit $6 \mathrm{ml} \mathrm{HCl}(0,2 \mathrm{~mol} / \mathrm{l})$ eluiert.

\section{Messung der Radioaktivität}

Die Radioaktivität wurde in 2 ml-Aliquoten (Eluate der Bio Rex 5-Säule sowie Durchlauf der Dowex Ag1-X2-Säule) bzw. in den Gesamteluaten nach Zusatz von $10 \mathrm{ml}$ Unisolve (Koch \& Light, Colnbrook, UK) in einem Liquid-Scintillationsspektrophotometer (SL 30 Intertechnique, Paris) gemessen.

Schema 2: Trennungsgang II zur Bestimmung der methylierten und konjugierten Noradrenalin-Mletaboliten.

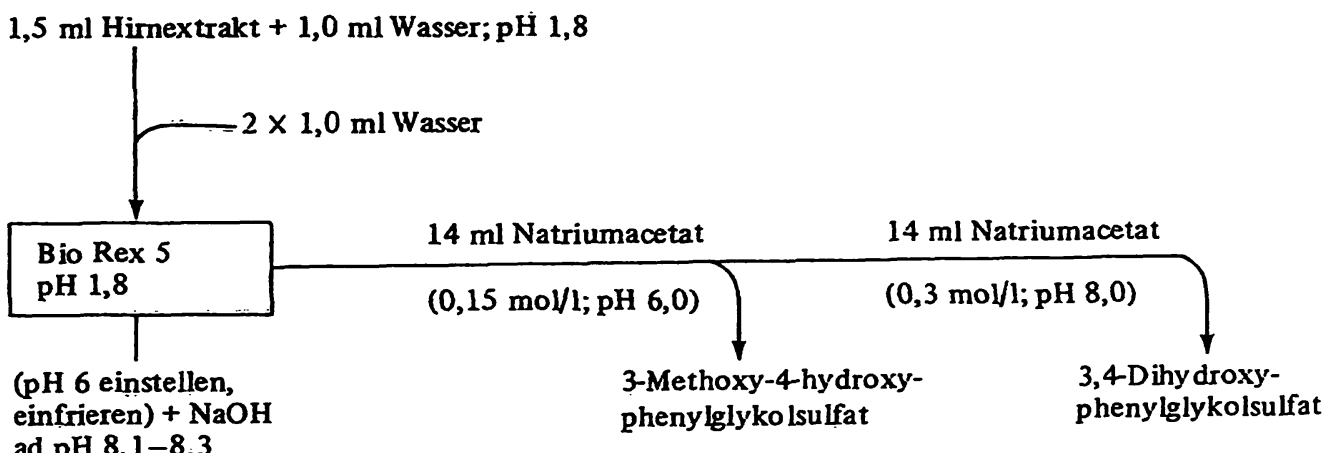

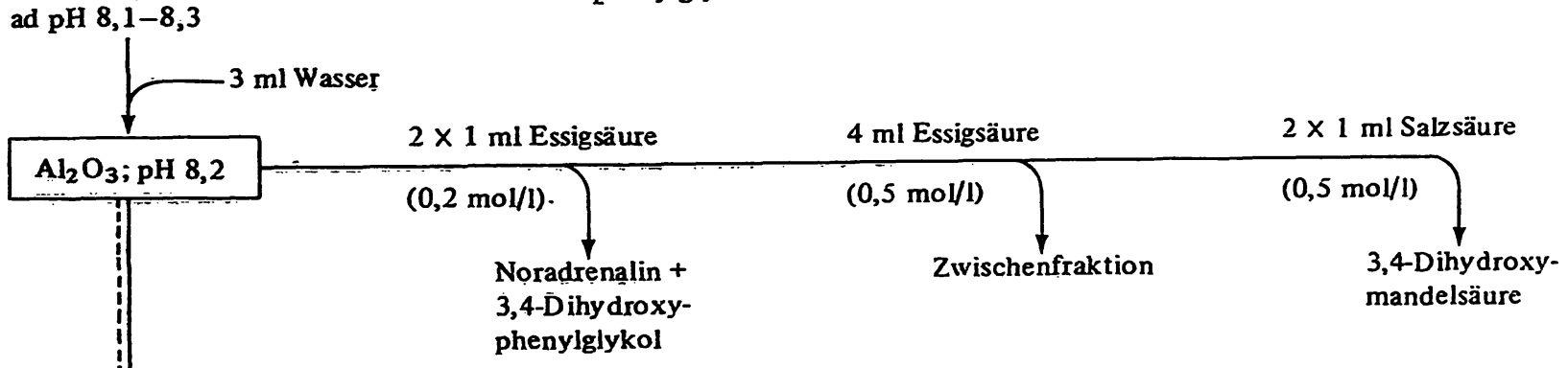

\begin{tabular}{|l|l|}
\hline $\begin{array}{l}\text { Dowex } 50 \mathrm{~W}-\mathrm{X} 4 \\
\text { pH 2,0 }\end{array}$ & $2 \times 1 \mathrm{ml}$ ethanol. Salz säure \\
\hline $\begin{array}{l}\text { AG 1-X2 } \\
\text { pH 4,5 }\end{array}$ & $2 \times 3 \mathrm{ml}$ \\
\hline 3-Methol/1; Ethanol $500 \mathrm{ml} / \mathrm{l})$
\end{tabular}

Vanillinmandelsäure 


\section{Berechnung der Ergebnisse}

Um methodisch bedingte Schwankungen der Radioaktivität auszugleichen, wurden die Anteile von Noradrenalin und seinen Metaboliten an der Gesamtradioaktivität in Prozenten angegeben. Die Halbwertszeiten wurden mit Hilfe der Kovarianzanalyse der zugrunde liegenden Regressionsgeraden statistisch miteinander verglichen.

Die Wirkung von Nembutal auf das Metabolitenmuster von Noradrenalin wurde mit der Varianzanalyse für faktorielle Versuchs anordnungen statistisch geprüft.

\section{Ergebnisse und Diskussion}

\section{Auftreninung der Metaboliten von Noradrenalin}

Zur Bestimmung von Noradrenalin und seinen Metaboliten aus Gewebeextrakten wurden zwei Trennuungsgänge durchgeführt. Die Auftrennung in einem einzigen Trennungsgang war mit den von uns gewählten Verfahren nicht möglich, weil einerseits die chemisch labilen Catechole zersetzt und andererseits bei Anwesenheit größerer Mengen von $\mathrm{Cl}^{-}$-Ionen die Glykolsulfate nicht mehr vollständig bei pH 1,8 an Bio Rex 5 gebunden werden. Im Trennungsgang I werden die Catechole im ersten Schritt an $\mathrm{Al}_{2} \mathrm{O}_{3}$ adsorbiert und so vor Oxidation geschützt; dabei werden folgende Verbindungen voneinander getrennt und bestimmt: Noradrenalin, 3,4Dihydroxymandelsäure, 3,4-Dihydroxyphenylglykol und Normetanephrin. Die restlichen Metaboliten, von denen die Glykolsulfate an Bio Rex 5 adsorbiert werden sollten, können nicht aus dem Durchlauf der Dowex 50W-X4-Säule bestimmt werden, weil zum Ansäuern der acetatgepufferten Lösung auf $\mathrm{pH} 1,8$ zuviel $\mathrm{HCl}$ nötig wäre. Darum werden im Trennungsgang II aus den essigsauren Proben nach $\mathrm{Zu}$ gabe geringer Mengen $\mathrm{HCl}$ im ersten Schritt die Glykolsulfate an Bio Rex 5 adsorbiert und anschließend der Trennungsgang I durchgeführt. Zuletzt wird Vanillinmandelsäure an Dowex AG1-X2 absorbiert. In diesem Trennungsgang werden 3-Methoxy-4-hydroxyphenylglykolsulfat, 3,4Dihydroxyphenylglykolsulfat, 3-Methoxy-4hydroxyphenylglykol und Vanillinmandel- säure bestimmt, nicht aber die Catechole, weil diese auf der Bio Rex 5-Säule teilweise zersetzt werden. Auch Normetanephrin kann in diesem Trennungsgang nicht bestimmt werden, weil die Noradrenalinartefakte fast ausschließlich in der Normetanephrinfraktion erscheinen. Die hier gewählte Kombination der Methode von Graefe et al. (4) (Trennungsgang I) mit der Vorschrift von Stone \& Mendlinger (8) (Adsorption an Bio Rex 5) erlaubt die vollständige Trennung vôn Noradrenalin und seinen Metaboliten aus Gehimextrakten. Sie ist relativ empfindlich, da - mit Ausnahme der Glykolsulfate die jeweils zu untersuchende Fraktion vollständig zur Messung der Radioaktivität eingesetzt werden kann. Die relativ gute Präzision ergibt șich daraus, dạß alle Metaboliten direkt und nicht auf Grund von Differenzmessungen bestimmt werden.

\section{Wiederfindungsversuche}

Die Ergebnisse der Wiederfindungsversuche sind in Tabelle 1 dargestellt. Sie liegen für die einzelnen Metaboliten zwischen 80 und $97 \%$. Geringe Anteile von Noradrenalin, von 3,4-Dihydroxyphenylglykol sowie von Vanillinmandelsäure finden sich in der 3,4-Dihydroxymandelsäure-Fraktion. Weil in Gewebeextrakten deutlich weniger 3,4-Dihydroxyphenylglykol und Vanillinmandelsäure vorkommen als 3,4-Dihydroxymandelsäure, können diese Verunreinigungen in der Regel vernachlässigt werden. Dagegen stört Noradrenalin wegen seiner größeren Konzentration im Gewebeextrakt die Bestimmung von 3,4-Dihydroxymandelsäure merklich. Dieser Fehler kann allerdings nach Bestimmung von Noradrenalin rechnerisch eliminiert werden. Die geringen Anteile von 3-Methoxy-4hydroxyphenylglykol, die in der Normetanephrin-Fraktion gefunden werden, machen sich wegen der geringen Menge des Glykols im Gewebeextrakt ebenfalls kaum störend bemerkbar. Unterwirft man radioaktiv markiertes Noradrenalin den Trennungsgängen I und II, so findet man - berechnet auf $100 \%$ Wiederfindung der Radioaktivität $-91,4 \%$ in der Noradre-

Tab. 1. Ergebnisse der Wiederfindungsversuche mit Noradrenalin und seinen Derivaten. Reinsubstanzen wurden dem im Text beschriebenen Trennungsgang unterworfen; die Wiederfindung in den einzelnen Fraktionen wurde in \% der eingesetzten Menge angegeben.

\begin{tabular}{|c|c|c|c|c|c|c|}
\hline \multirow[t]{2}{*}{ Substanz } & \multicolumn{6}{|l|}{ Fraktion } \\
\hline & $\begin{array}{l}\text { Nor- } \\
\text { adrenalin }\end{array}$ & $\begin{array}{l}\text { 3,4-Dihy droxy- } \\
\text { mandelsäure }\end{array}$ & $\begin{array}{l}\text { 3,4-Dihydroxy- } \\
\text { pheny lgly kol }\end{array}$ & $\begin{array}{l}\text { Normeta- } \\
\text { nephrin }\end{array}$ & $\begin{array}{l}\text { 3-Methoxy- } \\
\text { 4-hydroxy- } \\
\text { phenylglykol }\end{array}$ & $\begin{array}{l}\text { 3-Methoxy- } \\
\text { 4-hydroxy- } \\
\text { mandelsäure }\end{array}$ \\
\hline$\left[{ }^{3} \mathrm{H}\right]$ Noradrenalin & $91,4 \pm 2,3$ & $2,3 \pm 0,3$ & $0,6 \pm 0,1$ & $2,0 \pm 0,5$ & $3,1 \pm 1,1$ & $<0,1$ \\
\hline Noradrenalin & $80,7 \pm 3,2$ & $3,4 \pm 2,1$ & & & & \\
\hline 3,4-Dihy droxy mandelsäure & & $79,8 \pm 5,4$ & & & & \\
\hline 3,4-Dihy droxyphenylglykol & & $4,2 \pm 1,2$ & $83,2 \pm 3,3$ & & & \\
\hline Normetanephrin & & & & $96,6 \pm 4,0$ & & \\
\hline $\begin{array}{l}\text { 3-Methoxy-4hydroxy- } \\
\text { pheny lgly kol }\end{array}$ & & & & $2,2 \pm 1,0$ & $81,5 \pm 3,2$ & . \\
\hline $\begin{array}{l}\text { 3-Methoxy-4-hydroxy- } \\
\text { mandelsäure }\end{array}$ & & $5,6 \pm 0,8$ & & & & $80,0 \pm 6,1$ \\
\hline
\end{tabular}


nalin-Fraktion sowie zwischen 0,1 und $3,1 \%$ in den übrigen Fraktionen (vgl. Tab. 1). Das Auftreten dieser geringen Mengen an Radioaktivität in den verschiedenen Fraktionen ist zum Teil durch radioaktive Verunreinigungen des Ausgangsmaterials bedingt, die bis zu 5\% betragen und auch nach dünnschichtchromatographischer Reinigung kurzfristig wieder auftreten.

\section{Präzision der Methode}

Die Variationskoeffizienten für die Bestimmungen innerhalb der Serie sowie von Tag zu Tag sind in Tabelle 2 wiedergege ben. Genauigkeit und Empfindlichkeit der Methode reichten aus, um aus Extrakten von durchschnittlich $20 \mathrm{mg}$ Hypothalamusgewebe noch solche Metaboliten, die nur etwa 1-2\% der Radioaktivität enthielten, mit einem Variationskoeffizienten von $20 \%$ oder weniger $\mathrm{zu}$ bestimmen.

\section{Metabolitenmuster von $\left[{ }^{3} \mathrm{H}\right]$ Noradrenalin in verschiedenen Hirnregionen}

$1 \mathrm{~h}$ nach intraventrikulärer Injektion von $185 \mathrm{KBq}$ $D, L-\left[7-{ }^{3} \mathrm{H}\right]$ Noradrenalin wurden im gesamten Gehirn etwa 25-30\% der eingesetzten Radioaktivität wiedergefunden. Wie Tabelle 3 zeigt, erschienen davon 5,5\% im vorderen Hypothalamus, $12 \%$ in Pons und Medulla und zwischen $7 \%$ und $8,5 \%$ in den übrigen untersuchten Regionen. Etwa 50\% verblieben im Resthirn. Bezieht man die Radioaktivität auf Frischgewicht, so ergaben sich wie erwartet (9) - große regionale Unterschiede wegen der unterschiedlichen Dichte aminerger Nervenzellen.
Die spezifische Aktivität war besonders hoch in Septum und Hypothalamus ( 2 bis $3 \cdot 10^{6} \mathrm{Imp} . / \mathrm{min} \cdot \mathrm{g}$ ). Der niedrige Wert für Pons und Medulla $\left(0,4 \cdot 10^{6} \mathrm{Imp} . /\right.$ min $\cdot g$ ) erklärt sich aus der größeren Entfernung vom Injektionsort, denn Schanberg et al. (10) fanden bei intracisternaler Injektion deutlich höhere Werte.

Abbildung 1 zeigt die prozentuale Verteilung der Radioaktivität auf Noradrenalin und seine Metaboliten in den einzelnen Himregionen. Wie erwartet, wurde im Hypothalamus ein größerer Anteil von Noradrenalin gefunden als in Thalamus und Pons mit Medulla. Entsprechend fanden sich im Hypothalamus niedrigere Anteile der Metaboliten an der Radioaktivität als in Pons und Medulla. Hauptmetabolit war in allen Fällen 3-Methoxy-4hydroxyphenylglykolsulfat $(11,12)$. Die Aktivitätsanteile einiger Metaboliten wichen in einzelnen Regionen in typischer Weise von den Durchschnittswerten ab. Im hinteren Hypothalamus wurde relativ mehr 3-Methoxy4-hydroxyphenylglykolsulfat gebildet als in anderen Regionen. In Pons und Medulla wurde ein größerer Anteil von unkonjugiertem 3-Methoxy-4-hydroxyphenylglykol gefunden. Im Septum entstand mehr Normetanephrin und weniger 3,4-Dihydroxyphenylglykolsulfat als in den übrigen Regionen. Bei der Bestimmung aller Metaboliten von Noradrenalin werden demnach die regionalen Unterschiede im Stoffwechsel von Noradrenalin deutlicher als mit früher üblichen Bestimmungsmethoden, bei denen die Gruppe der methylierten und der nichtmethylierten Desaminierungsprodukte nicht weiter aufgetrennt werden (13).

Tab. 2. Reproduzierbarkeit der Bestimmungen von Noradrenalin und seinen Metaboliten in den Trennungsgängen l (für Catechole und Normetanephrin) und II (für methylierte und konjugierte Metaboliten).

Die Zahlen geben die Variationskoeffizienten (\%) bei Mehrfachbestimmungen aus einer Probc an.

\begin{tabular}{|c|c|c|c|c|c|c|c|c|}
\hline $\begin{array}{l}\text { Experimentelle } \\
\text { Bèdingungen }\end{array}$ & $\begin{array}{l}\text { Nor- } \\
\text { adrenalin }\end{array}$ & $\begin{array}{l}\text { Normeta- } \\
\text { nephrin }\end{array}$ & $\begin{array}{l}3,4- \\
\text { Dihy droxy- } \\
\text { mandelsäure }\end{array}$ & $\begin{array}{l}\text { Vanillin- } \\
\text { mandelsäure }\end{array}$ & $\begin{array}{l}3,4- \\
\text { Dihydroxy- } \\
\text { pheny } 1- \\
\text { glykol }\end{array}$ & $\begin{array}{l}\text { 3-Methoxy- } \\
\text { 4-hydroxy- } \\
\text { phenyt } \\
\text { glykol }\end{array}$ & $\begin{array}{l}\text { 3,4- } \\
\text { Dihydroxy- } \\
\text { phenyl- } \\
\text { glykolsulfat }\end{array}$ & $\begin{array}{l}\text { 3-Methoxy- } \\
\text { 4-hydroxy- } \\
\text { phenyt } \\
\text { glykolsulfat }\end{array}$ \\
\hline $\begin{array}{l}\text { Bestimminugen } \\
\text { am selben Tag }\end{array}$ & 4,1 & 3,9 & 6,0 & 6,2 & 5,2 & 2,2 & 3,1 & 1,2 \\
\hline $\begin{array}{l}\text { Bestimmungen } \\
\text { an verischiẹdenen } \\
\text { Tagen }\end{array}$ & 4,7 & $5,4^{\circ}$ & 6,4 & 9,0 & 8,5 & 3,7 & 5,4 & 4,0 \\
\hline
\end{tabular}

Tab. 3. Verteilung der wiedergefundenen Radioaktivität in \% sowie auf Frischgewicht bezogen in verschiedenen Hirnregionen nach Injektion von $\left[{ }^{3} \mathrm{H}\right]$ Norạdrenalin.

Weitere Einzelheiten, vgl. Text. Die Zahlen in der Tabelle sind Mittelwerte aus mindestens 8 Einzelbestimmungen.

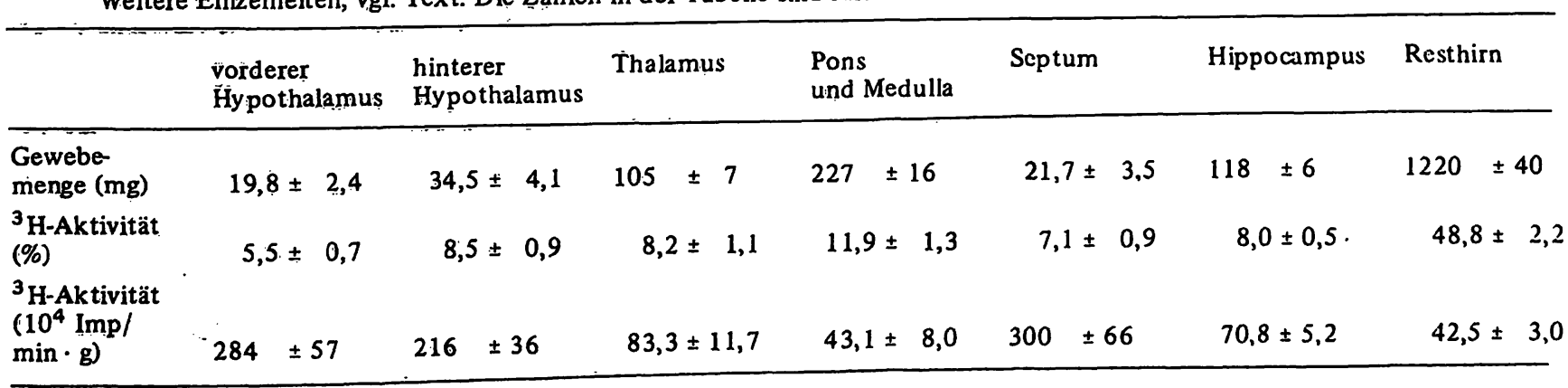


Wirkung einer Nembutalnarkose auf die Gesamtaktivität in Gehirn und Serum nach Injektion von $\left[{ }^{3} \mathrm{H}\right]$ Noradrenalin

Nembutal beeinflußt die Umsatzgeschwindigkeit von Neurotransmittern (5). Wie Abbildung 2 zeigt, nahm $\mathrm{zw}$ ischen $1 \mathrm{~h}$ und $4 \mathrm{~h}$ nach intraventrikulärer Injektion von $\left[{ }^{3} \mathrm{H}\right]$ Noradrenalin die Radioaktivität im Gehirn bei halblogarithmischer Darstellung linear mit der Zeit ab. Die Halbwertszeit betrug nach Injektion in Äthernarkose 3,3 h. In Nembutalnarkose wurde stets eine höhere Radioaktivität gefunden, die mit etwa doppelt so langer $\mathrm{Halb}$ wertszeit abnahm ( $t_{1 / 2}$ etwa $6 \mathrm{~h}$ ) wie in der Vergleichsgruppe $(p<0,05)$. Im Gegensatz zur Radioaktivität im Gehirn war die Radioaktivität im Serum nach Äthernarkose höher als in Nembutalnarkose ( $p<0,02$ zum Zeitpunkt $t=1 \mathrm{~h}$ ). Die Radioaktivität im Serum nahm im Wachzustand noch schneller $\left(t_{1 / 2}=1,8 \mathrm{~h}\right)$, in Nembutalnarkose dagegen noch langsamer $\left(t_{1 / 2}=7,5 \mathrm{~h}\right) \mathrm{ab}$ als die Radioaktivität im Gehirn. Der Unterschied zwischen den Behandlungsgruppen war hochsignifikant $(p<0,001)$. In der Vergleichsgruppe wird also die Radioaktivität relativ schnell aus dem Gehirn ans Blut
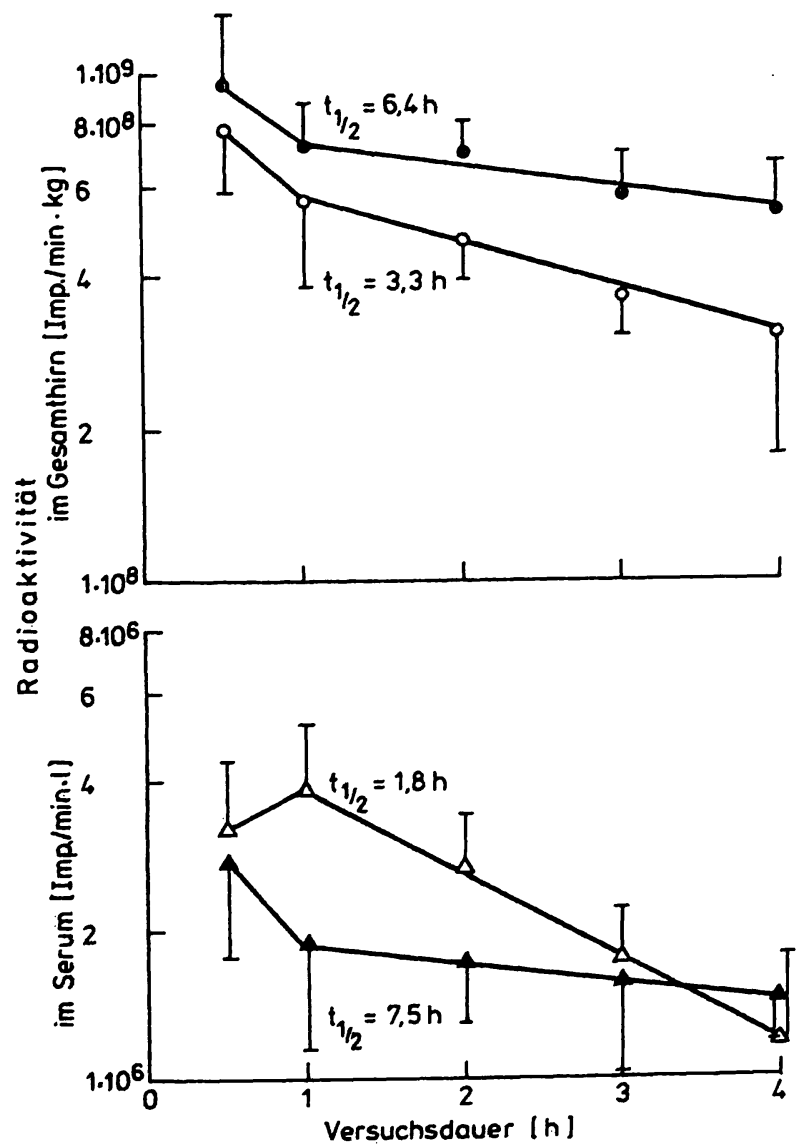

Abb. 2. Abhängigkeit der Radioaktivität von der Zeit im Gesamtgehirn und im Serum nach intraventrikulärer Injektion von $l^{3} \mathrm{H}$ Noradrenalin. Angegeben sind Mittelwerte aus 4-7 Einzelbestimmungen \pm Stand ardabweichungen. $0, \Delta=$ Injektion in Äthernarkose

$\bullet, \Delta=$ Injektion in Nembutalnarkose

$t_{1 / 2}=$ Halbwertszeit. abgegeben und noch schneller aus dem Blut eliminiert. In Nembutalnarkose dagegen scheint die periphere Eliminierung der markierten Produkte noch mehr verlangsamt zu sein, als die Abgabe aus dem Gehirn an das Blut.

\section{Wirkung einer Nembutalnarkose auf dic Halbwertszeit von Noradrenalin in verschiedenen Hirnregionen}

Im Hypothalamus, im Thalamus und in Pons und Medulla nahm die Menge von radioaktivem Noradrenalin bei logarithmischer Auftragung zwischen 0,5 und $4 \mathrm{~h}$ nach der Injektion linear mit der Zeit ab (Abb. 3). Dabei waren die Halbwertszeiten in Nembutalnarkose (6,5 bis $9,4 \mathrm{~h}$ ) deutlich länger als nach $\AA$ thernarkose $(2,7$ bis $3,1 \mathrm{~h})(\mathrm{p}<0,01)$. Die für die Vergleichsgruppe bestimmten Halbwertszeiten entsprechen Angaben aus der Literatur $(14 ; 15)$. Dagegen fanden andere Autoren eine barbituratbedingte Verzögerung des Umsatzes von Noradrenalin nur im Cortex, nicht aber auch im Hypothalamus, Thalamus und Pons und Medulla (16).

Bei Extrapolation der Abklingkurven auf $t=0$ fanden sich keine Unterschiede der Primäraufnahme von Noradrenalin in Nembutal- oder Äthernarkose. Nembutal scheint also in vivo ebenso wie in vitro die Aufnahme von Noradrenalin nicht zu beeinflussen (17).

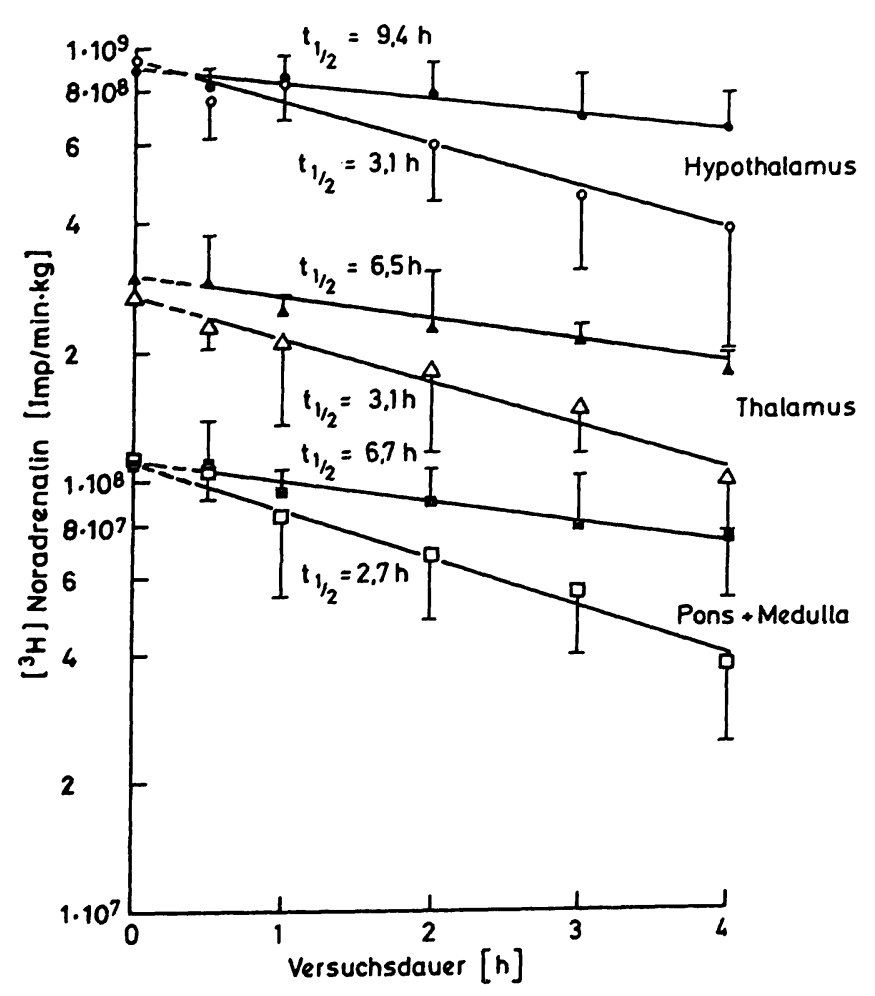

Abb. 3. Abhängigkeit der Menge von wiedergefundenem Noradrenalin von der Zeit in verschiedenen Hirnregionen nach intraventrikulärer Injektion von $l^{3} \mathrm{H}$ J Noradrenitlin. Angegeben sind Mittelwerte aus 4-7 Einzelbestimmungen \pm Stand ardabweichungen. $0, \Delta, 0=$ Injcktion in Äthernarkose $\bullet, \triangle,=$ = Injektion in Nembutalnarkose $t_{1 / 2}=$ Halbwertszeit 


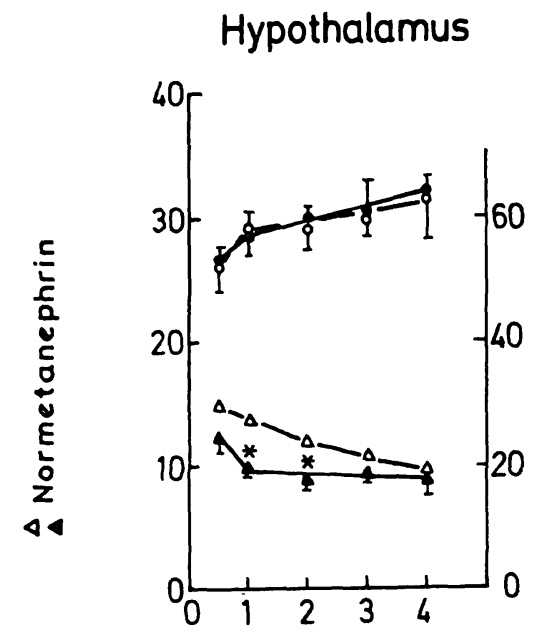

\section{Thalamus}
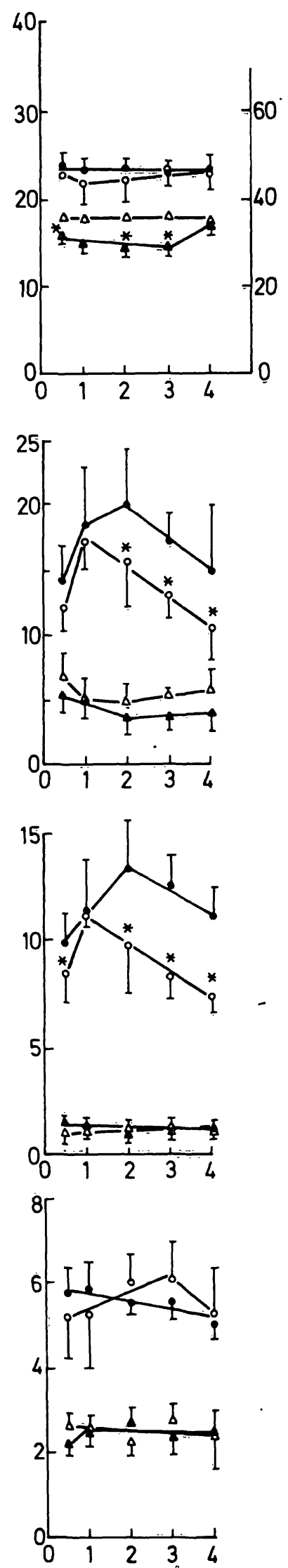

Pons+ Medulla
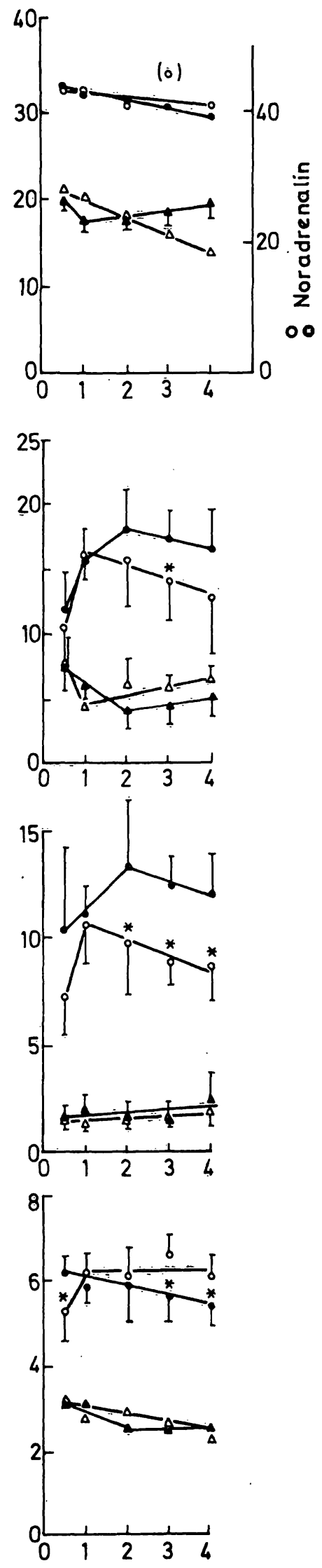

Zeit nach Injektion von $\left[{ }^{3} \mathrm{H}\right]$ Noradrenalin $[\mathrm{h}]$

Abb. 4. Anteile von Noradrenalin und seinen Metaboliten an der wiedergefundenen Radioaktivität in \% nach intraventrikulärer Injektion von $\left[{ }^{3} \mathrm{H}\right]$ Noradrenalin und deren Abhängigkeit von der Versuchsdauer in verschiedenen Hirnregionen. Angegeben sind Mittelwerte aus 4-7 Einzelbestimmungen \pm Standardabweichungen.

$\circ, \Delta=$ Injektion in Äthernarkose $\quad \bullet, \Delta=$ Injektion in Nembutalnarkose $\quad *=p<0,05$ 


\section{Wirkung einer Nembutalnarkose auf das Metaboliten- muster von Noradrenalin in verschiedenen Hirnregionen}

Wie Abbildung 4 zeigt, nimmt im Hypothalamus der prozentuale Anteil von Noradrenalin an der Radioaktivität mit der Zeit zu $(p<0,02)$, während er im Thalamus unverändert bleibt und in Pons und Medulla abnimmt $(p<0,05)$. Die Zunahme im Hypothalamus kann durch die Aufnahme von Noradrenalin in den Pool mit längerer Halbwertszeit erklärt werden (18). Die Nembutalnarkose hat keinen Einfluß auf diese Vorgänge. In den drei untersuchten Regionen befinden sich in beiden Versuchsgruppen jeweils gleiche Anteile der ${ }^{3} \mathrm{H}$-Aktivität in der Noradrenalin-Fraktion, d.h. bei der erhöhten Gesamtaktivität ist sowohl die Menge an Noradrenalin als auch diejenige der Abbauprodukte in Nembutalnarkose höher als in der Vergleichsgruppe. Obwohl also weniger Noradrenalin freigesetzt wird und enzymatisch abgebaut werden muß, findet man eine größere Menge an Metaboliten. Das deutet auf einen verzögerten Abtransport der Abbauprodukte hin.

Abbildung 4 zeigt weiter, daß sich die Nembutalnarkose deutlich auf die Bildung der Sulfate der beiden Glykole sowie auf die Bildung von Normetanephrin auswirkt. In Hypothalamus und Thalamus werden in Nembutalnarkose geringere Anteile von Normetanephrin gefunden als nach Athernarkose $(p<0,01)$. Ebenso wird in der Nembutalgruppe durchweg weniger freies 3-Methoxy-4 hydroxyphenylglykol gebildet als in der Kontrollgruppe ( $p<0,05$; in Hypothalamus $p<0,01)$. Das Umgekehrte gilt für die Glykolsulfate: Hier liegen die Werte in allen untersuchten Regionen in Nembutalnarkose deutlich höher $(p<0,005)$. Die Glykolsulfate zeigen auch die deutlichsten Veränderungen mit der Zeit: Ihre Aktivität durchläuft - wie auch von Nielsen (19) beobachtet $-1 \mathrm{~h}$ nach der Injektion von Noradrenalin in Äthernarkose ein Maximum, während in Nembutalnarkose dieses Maximum in Thalamus und Pons und Medulla erst $2 \mathrm{~h}$ nach der Injektion erreicht wird. Die übrigen Metaboliten zeigten keine oder nur uncharakteristische Veränderungen.

\section{Literatur}

1. Brever, H. \& Köster, G. (1975) Adv. Biosci. 15, 287-300.

2. Breuer, H., Köster, G., Schneider, H.-T. \& Ladosky, W. (1978) in: Brain=Endocrine-Interactions III Neural Hormones and Reproduction (Scott, D. E., Kozlowski, G. P. \& Weindl, A., eds.) Karger/Basel, 274-285.

3. Breuer, H. \& Köster, G. (1974) J. Steroid Biochem. 5, 961967.

4. Graefe, K. H., Stefano, F. J. E. \& Langer, S. Z. (1973) Biochem. Pharmacol 22, 1147-1160.

5. Corrodi, H., Fuxe, K. \& Hökfeld, T. (1966) J. Pharm. Pharmacol 18, 556-558.

6. Noble, E. P., Wurtman, R. W. \& Axelrod, J. (1967) Life Sci. 6, 281-291.

7. Anton, A. H. \& Sayre, D. F. (1962) J. Pharmacol. Exp. Ther. $138,360-375$.

8. Stone, E. A. \& Mendlinger, S. (1974) Anal: Biochem. 62, 592-5.97.
Die niedrigeren Werte für Normetanephrin in der Nembutalgruppe korrespondieren mit dem langsameren Umsatz von Noradrenalin und können wie dieser durch eine geringere Nervenaktivität bedingt sein (20). Die unter Nembutal erhöhten Werte für die Glykolsulfate zusammen mit der erniedrigten Radioaktivität im Serum deuten dagegen auf eine gestörte Abgabe der Endabbauprodukte aus dem Gehirn ins Blut hin. Der Rückstau der Endabbauprodukte scheint dabei nicht allein durch die herabgesetzte Gehirndurchblutung bedingt zu sein; während in der Äthergruppe die Aktivitäten der beiden Glykolsulfate mit der Radioaktivität im Serum positiv korrelieren $(p<0,025$ für Thalamus und $p<0,001$ für Hypothalamus), korrelierten die Aktivitätskurven unter der Wirkung von Nembutal nicht mehr miteinander.

\section{Schlußfolgerungen}

Die hier beschriebene Methode zur Bestimmung von $\left[{ }^{3} \mathrm{H}\right]$ Noradrenalin und seinen Metaboliten aus Himgewebe ist nach unserer Erfahrung anderen Verfahren wegen ihrer Empfindlichkeit, Reproduzierbarkeit und Praktikabilität überlegen. Mit ihrer Hilfe können äußere Einflüsse auf den gesamten Stoffwechsel von Noradrenalin im Gehirn untersucht werden. Die vorgelegten Ergebnisse zeigen, daß eine Nembutalnarkose in Hypothalamus, Thalamus und Pons und Medulla nicht nur die Halbwertszeit von Noradrenalin verlängert, sondern auch das Metabolitenmuster verändert und die Abgabe der Metaboliten vom Gehirn an das Blut verzögert. Für in vivo-Stoffwechselversuche mit Noradrenalin scheint Nembutal als Anästhetikum weniger geeignet zu sein als Ä ther - nicht nur, weil die Narkose länger anhält und Gehirndurchblutung wie Nervenaktivität herabgesetzt werden, sondern weil darüber hinaus weitere Störungen auftreten. Es bleibt offen, ob alle Änderungen durch Membraneffekte zu erklären sind oder ob Nembutal zusätzlich eine direkte Wirkung auf den enzymatischen Abbau von Noradrenalin ausübt.
9. Glowinsky, J. \& Iversen, L. L. (1966) J. Neurochem. 13, 655-669.

10. Schanberg, S. M., Schildkraut, J. J. \& Kopin, I. J. (1967) J. Pharmacol Exp. Ther. 157, 311-318.

11. Schanberg, S. M., Schildkraut, J. J., Breese, G. R. \& Kopin, I. J. (1968) Biochem. Pharmacol. 17, 247-254.

12. Sugden, R. F. \& Eccleston, D. (1971) J. Ncurochem. 18, 2461-2468.

13. Glowinsky, J., Axelrod, J. \& Iversen, L. L. (1966) J. Pharmacol. Exp. Ther. 153, 30-41.

14. Iversen, L. L. \& Glowinsky, J. (1966) J. Neurochem. 13, 671-682.

15. Taylor, K. M. \& Laverty, R. (1969) J. Neurochem. 16, 1367-1376.

16. Lidbrink, P., Corrodi, H., Fuxe, K. \& Olson, L. (1972) Brain Res. 45, 507-524. 
17. Lidbrink, P. \& Farnebo, L.-O. (1973) Neuropharmacology $12,1087-1095$.

18. Glowinsky, J., Kopin, I. J. \& Axelrod, J. (1965) J. Neurochem. $12,25-30$.
19. Nielsen, M. (1976) J. Neurochem. 27, 493-500.

20. Stolk, J. M., Conner, R. L., Levine, S. \& Barchas, J. D. (1974) J. Pharmacol. Exp. Ther. 190, 193-209.

Prof. Dr. Heinz Breuer

Institut für Klinische Biochemie der Universität

Venusberg

5300 Bonn 1 